\title{
Ventricular diverticulum
}

\author{
SUSUMU WAKAI, MASAKATSU NAGAI \\ From the Department of Neurosurgery, Dokkyo University School of Medicine, Tochigi, Japan
}

SUMMARY A ventricular diverticulum was found on computed tomography in six out of 25 cases of congenital obstructive hydrocephalus. In all six cases, the diverticulum was located on the medial wall of the trigone. In three cases, it was restricted to the tentorial hiatus with a recognisable superior cerebellar cistern (small). In two cases, it occupied the tentorial hiatus without a recognisable cistern (medium). The remaining case had a diverticulum extending into the posterior fossa that caused cerebellar ataxia (large). In all patients, the diverticulum regressed after ventriculo-peritoneal shunt. The characteristics of the computed tomograms, the clinical significance and the pathogenesis of this phenomenon are discussed.

Spontaneous ventriculocisternostomy or diverticular formation in advanced obstructive hydrocephalus is well-known ${ }^{i-3}$ following DeLange, ${ }^{4}$ and Penfield' $s^{5}$ descriptions in 1929. In a previous report, ${ }^{6}$ the authors presented a case of ventricular diverticulum causing cerebellar ataxia, which was shown to be a cystic mass at the tentorial hiatus as well as in the posterior fossa by computed tomography (CT). No report regarding the incidence, characteristics of the CT picture, and clinical significance of diverticular formation has been available to date. The present report is of six examples of this condition among cases of congenital hydrocephalus observed during the past six years.

\section{Clinical materials and methods}

The clinical material consisted of 25 cases of congential obstructive hydrocephalus treated in our institute during the past six years, from June 1977 to May 1983. All patient records and CT scans were reviewed to determine the presence, location and size of the ventricular diverticulum and clinical symptoms related to this phenomenon. The causes of hydrocephalus in all 25 patients are shown in table 1.

Summary of cases (table 2)

A ventricular diverticulum was found in six of the 25 cases $(24 \%)$. There were two males and four females, whose age ranged from two days to 11 years.

Causes of hydrocephalus There were four aqueductal

Address for reprint requests: Susumu Wakai, MD, Section of Neurocytology LNNS, NINCDS, National Institutes of Health, Bldg 36, Bethesda, MD 20205, USA.

Received 7 July 1983.

Accepted 18 November 1983 stenoses, including one with a myelomeningocele. The remaining two were porencephaly and occlusion of the outlet of the fourth ventricle.

Site of ventricular diverticulum In all six cases, the ventricular diverticulum was located on the medial wall of the trigone of the lateral ventricle: five on the left and one on the right. All but one were on the side with the larger trigone. In the case with porencephaly, the ventricular diverticulum was present on the smaller side of the trigone, which was the same side as that of porencephaly. The aperture of the ventricular diverticulum was clearly located at the medial wall of the trigone (fig 1-4).

Size of the ventricular diverticulum In three cases, the ventricular diverticulum was restricted to the tentorial hiatus and the superior cerebellar cistern was recognised partially in the contralateral side to ventricular diverticulum. (small, fig 1). In two cases, the ventricular diverticulum occupied the tentorial hiatus without a recognisable cistern (medium, figs 2 and 3 ). The remaining one (case 6) had a ventricular diverticulum extending into the posterior fossa through the tentorial hiatus. (large, fig 4).

Symptoms and signs related to ventricular diverticulum The only case with a large ventricular diver-

Table 1 Causes of Hydrocephalus

\begin{tabular}{lll}
\hline Aqueduct stenosis & 9 (3) \\
Aqueduct stenosis with myelomeningocele & 8 (1) \\
Holoprocencephaly (semilobar type) & $3^{*}$ \\
Dandy-Walker syndrome & 3 \\
Occlusion of the outlet of the IVth ventricle & 1 (1) \\
Porencephaly & 1 (1)
\end{tabular}

Number in parenthesis indicates number of the case with diverticular formation in the lateral ventricle.

${ }^{*}$ A case associated with the Dandy-Walker syndrome is included. 

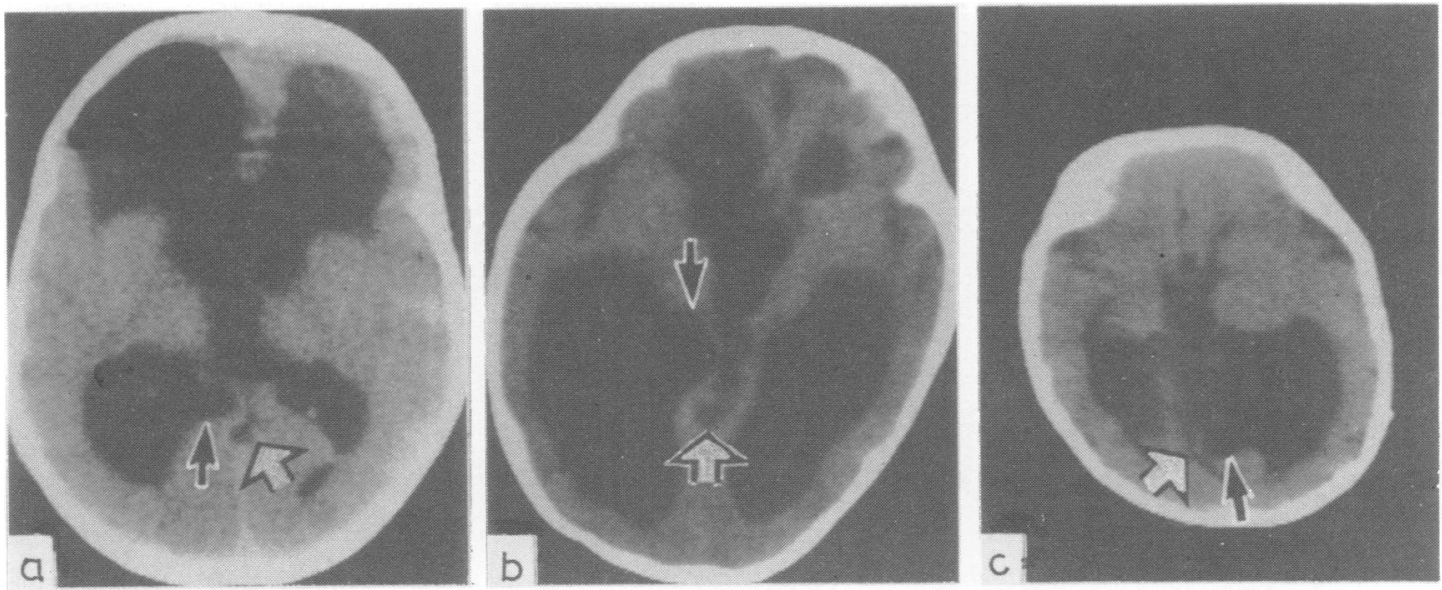

Fig 1 Computed tomography scans without contrast enhancement in cases with small ventricular diverticulum (VD). (A) Case 1. Aqueduct stenosis. (B) Case 2. Occlusion of the outlet of the fourth ventricle. (C) Case 3. Aqueduct stenosis with myelomeningocele. Black arrow indicates ventricular diverticulum in each picture. Superior cerebellar cistern (open arrow) is partially recognized in all three cases.
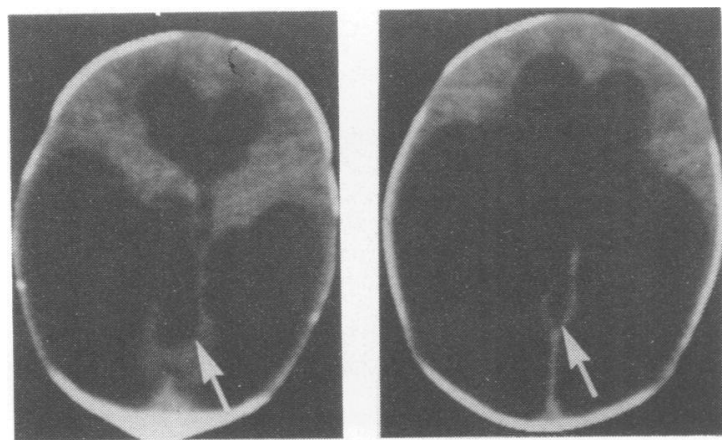

Fig 2 Computed tomography scans without contrast enhancement in case 4 (aqueduct stenosis). Medium-sized ventricular diverticulum is seen in the medial wall of the trigone (arrows). It occupies the tentorial hiatus without recognisable cistern. ticulum (case 6) showed symptoms secondary to ventricular diverticulum. This patient had cerebellar ataxia, which disappeared a few days after ventriculo-peritoneal shunt.

Operation and course Five cases underwent ventricular peritoneal shunt. Suboccipital craniectomy was carried out in the last (case 2) to open the occlusion of the outlet of the fourth ventricle followed by shunt. Metrizamide CT ventriculography was undertaken in two cases (cases 5 and 6) after surgery. The ventricular diverticulum was clearly observed in both cases (fig 3, B). In all cases, the ventricular diverticulum regressed after operation. In three patients with medium or large-sized ventricular diverticulum, the superior cerebellar cistern was visible on subsequent CTs following the shunt (figs 3, C and 4, D-F).

Three children (cases 2, 3, and 4) were bed-bound for 4 to 5 years after operation. One died two and half years after the operation due to shunt obstruction. Case 5 is surviving in good functional state. Case 6 is also doing well but is attending a school for the blind.

Table 2 Summary of cases with ventricular diverticulum

\begin{tabular}{|c|c|c|c|c|c|c|c|}
\hline $\begin{array}{l}\text { Case } \\
\text { No. }\end{array}$ & Age & Sex & Cause of hydrocephalus & Size of $V$ & Site of $V D$ & $\begin{array}{l}\text { Symptoms related } \\
\text { to } V D\end{array}$ & Operation \\
\hline $\begin{array}{l}1 \\
2\end{array}$ & $\begin{array}{l}4 \mathrm{mo} \\
8 \mathrm{yr}\end{array}$ & $\begin{array}{l}\mathbf{F} \\
\mathbf{M}\end{array}$ & $\begin{array}{l}\text { Aqueductal stenosis } \\
\text { Occlusion of the small outlet of the } \\
\text { 4th ventricle }\end{array}$ & $\begin{array}{l}\text { small } \\
\text { small }\end{array}$ & $\begin{array}{l}\text { Lt. LV } \\
\text { Lt. LV }\end{array}$ & $(-)$ & $\begin{array}{l}\text { V-P shunt } \\
\text { Suboccipital craniectomy and } \\
\text { V-P shunt }\end{array}$ \\
\hline 3 & 2 day & $\mathbf{F}$ & $\begin{array}{l}\text { Aqueductal stenosis with } \\
\text { myelomeningocele }\end{array}$ & small & Rt. LV & $(-)$ & V-P shunt \\
\hline $\begin{array}{l}4 \\
5 \\
6\end{array}$ & $\begin{array}{l}8 \text { day } \\
1 \mathrm{mo} \\
11 \mathrm{yr}\end{array}$ & $\begin{array}{l}\mathbf{F} \\
\mathbf{M} \\
\mathbf{F}\end{array}$ & $\begin{array}{l}\text { Aqueductal stenosis } \\
\text { Porencephaly } \\
\text { Aqueductal stenosis }\end{array}$ & $\begin{array}{l}\text { medium } \\
\text { medium } \\
\text { large }\end{array}$ & $\begin{array}{l}\text { Lt. LV } \\
\text { Lt. LV } \\
\text { Lt. LV }\end{array}$ & $\begin{array}{l}(-) \\
\text { Cerebellar ataxia }\end{array}$ & $\begin{array}{l}\text { V-P shunt } \\
\text { V-P shunt } \\
\text { V-P shunt }\end{array}$ \\
\hline
\end{tabular}

VD: ventricular diverticulum, LV: lateral ventricle, V-P shunt: ventriculo-peritoneal shunt. 

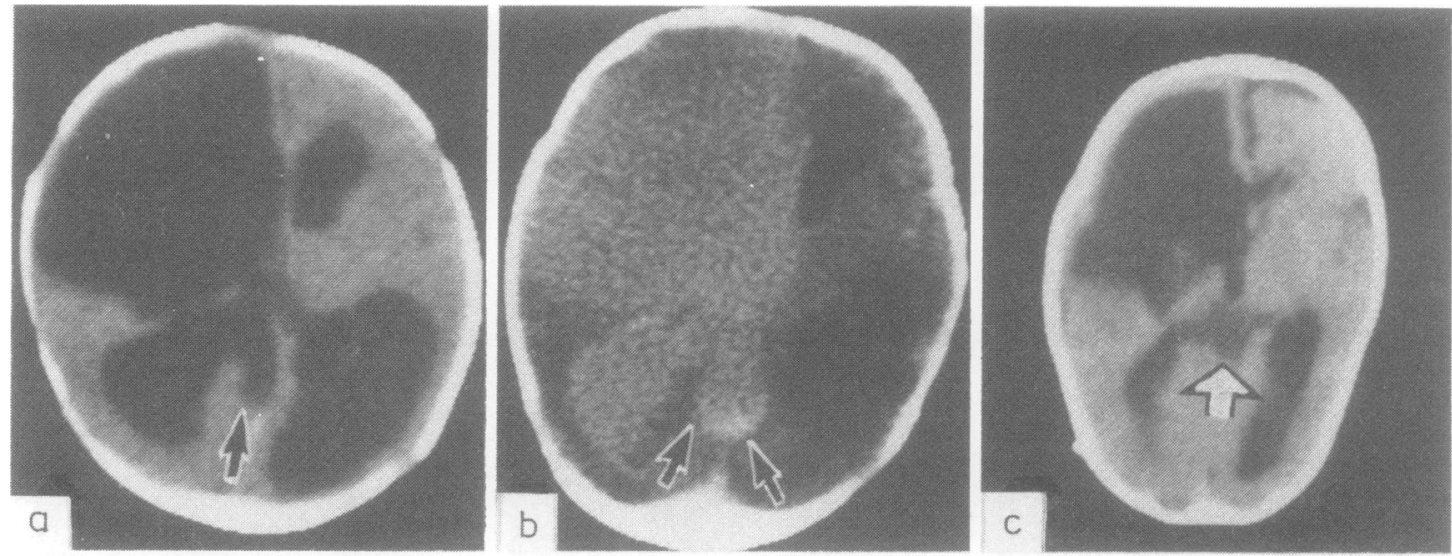

Fig 3 Computed tomography scans of the patient with porencephaly (case 5). (A) Without contrast enhancement. Arrow indicates ventricular diverticulum (medium) which occupies the tentorial hiatus. Superior cerebellar cistern is not discernible. (B) Metrizamide CT ventriculography after ventriculo-peritoneal shunt. Diverticulum is clearly visible. (C) Computed tomography one year after shunt. Diverticulum regresses. Arrow indicates superior cerebellar cistern.
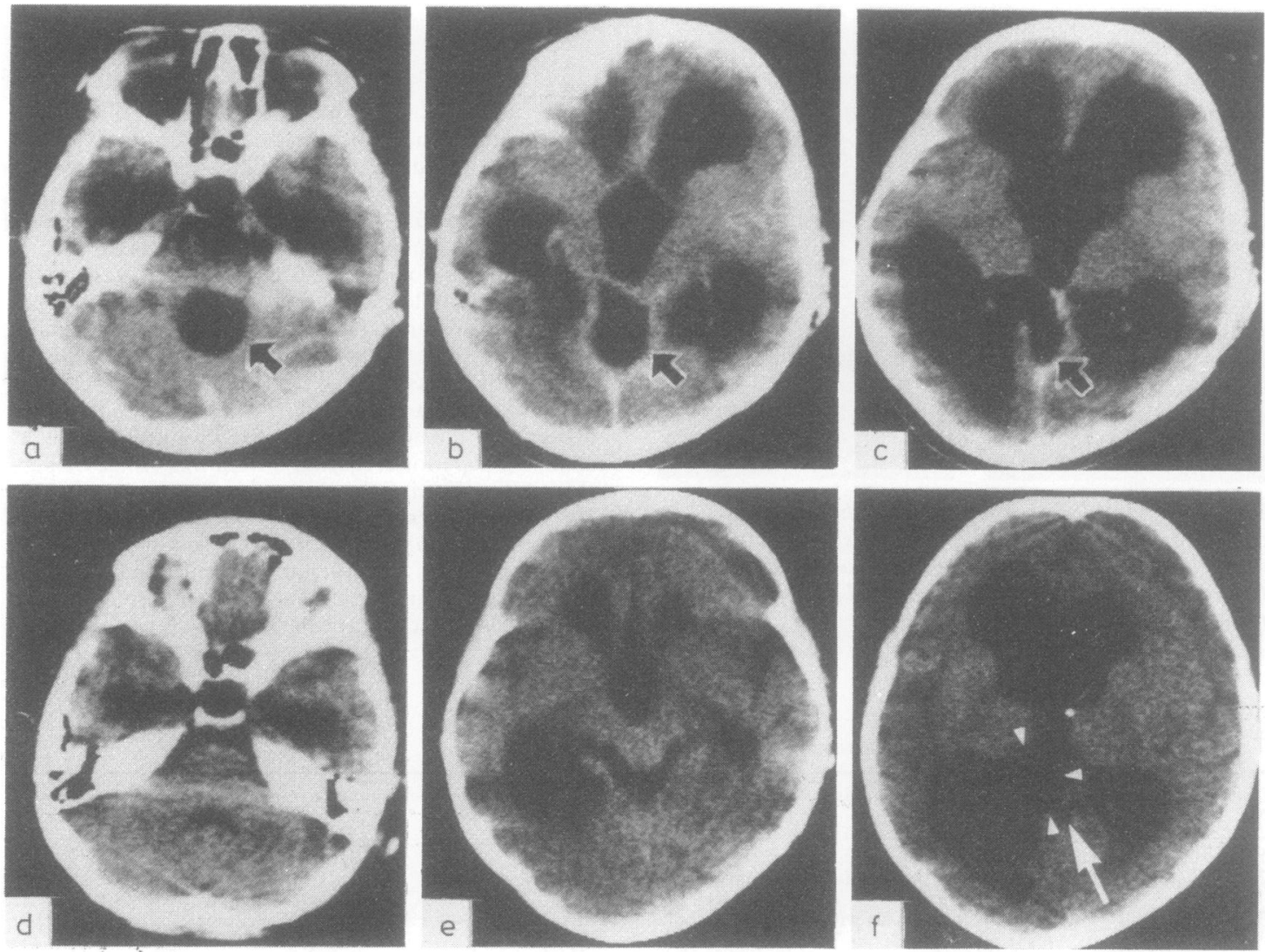

Fig 4 Computed tomography scans in case 6 (aqueduct stenosis). (A-C) With contrast enhancement. A large ventricular diverticulum is seen in the medial wall of the trigone (arrow in $C$ ) extending through the tentorial hiatus (arrow in $B$ ) into the posterior fossa (arrow in $A$ ). (D-F:) Computed tomography without contrast enhancement taken two weeks after ventriculo-peritoneal shunt. The fourth ventricle, which is normal in size and shape, is seen in $D$. The quadrigeminal (E) and the superior cerebellar cistern (arrow in F) are recognized. Arrow heads in F indicate a regressed diverticulum. 


\section{Discussion}

There has been no report on the incidence of ventricular diverticulum in cases of congenital hydrocephalus. Our present study revealed that in six out of 25 such cases (24\%) a diverticulum was found in the medial wall of the trigone on the initial CT scan.

We classified the ventricular diverticulum observed at this site into three types according to size and relationship to the tentorial hiatus and the superior cerebellar cistern. Cases with small and medium-sized ventricular diverticulum showed no symptoms related to it. Only one case with a large ventricular diverticulum developed cerebellar ataxia secondary to this phenomenon. Among the cases of large-sized ventricular diverticulum reported in the literature, only a few cases have shown clinically overt cerebellar ataxia. ${ }^{7-13}$ Medium or large-sized ventricular diverticulum without direct compression symptoms may also have a clinical significance in the compression of the aqueduct of Sylvius which results in exacerbation of hydrocephalus. ${ }^{14}$

According to the review of the literature in our previous article, ${ }^{6}$ a ventricular diverticulum was located mainly in the medial wall of the trigone as well as in the posterior wall of the third ventricle. In the present series, the ventricular diverticulum of all six cases was in the medial wall of the trigone. There was no case in which the ventricular diverticulum formed in the posterior wall of the third ventricle or in other sites. There can be little doubt that increased intraventricular pressure played an important role in the pathogenesis of the diverticular formation. Under such circumstances, a ventricular diverticulum may form at the weakest point of the ventricular wall. Anatomically, such a point is the medial wall of the trigone, which lies between the forward-sweeping crus of the fornix and the forceps major.9 ' ${ }^{15}$ Childe and McNaughton" noted thinning out and hollowing at just this portion of the trigone in several brains with marked hydrocephalus.

Cystic lesions, observed near the tentorial hiatus and the superior cerebellar cistern, which should be considered in differential diagnosis of the ventricular diverticulum formed in the medial wall of the trigone, are: (1) arachnoid cyst, (2) cystic tumour arising from the pineal gland or its surrounding structures, (3) dorsal sac of holoprosencephaly, (4) ventricular diverticulum in the posterior wall of the third ventricle. If one keeps in mind that a ventricular diverticulum at this site has an aperture in the medial wall of the trigone, the correct diagnosis can easily be made.

\section{Addendum}

After submission of this paper for publication, we found an article by Naidich TP, McLone DG, Hahn YS, Hanaway J (Atrial diverticula in severe hydrocephalus. $A J N R$ 1982;3:257-66). They demonstrated ventricular diverticula in $25 \%$ of patients with advanced hydrocephalus, which coincides with the incidence in the current series. They elaborately described the CT criteria for this entity. We apologise for an inadvertent omission of their article in the references.

\section{References}

' Milhorat TH. Hydrocephalus and the cerebrospinal fluid. Baltimore: Williams \& Wilkins, 1972;113-5.

${ }^{2}$ Harwood-Nash DC, Fitz CR. Neuroradiology in Infants and Children. Vol. 3. St Louis: Mosby, 1976;967-8.

${ }^{3}$ Zülch KJ. Atlas of Gross Neurosurgical Pathology. Heidelberg: Springer, 1975;209-19.

${ }^{4}$ de Lange C. Spontaneous healing in a case of hydrocephalus. Proc Royal Acad Amst 1929;32:78-85.

${ }^{5}$ Penfield W. Diencephalic autonomic epilepsy. Arch Neurol Psychiat 1929;22:358-74.

- Wakai S, Narita J, Hashimoto K, Nagai M. Diverticulum of the lateral ventricle causing cerebellar ataxia. Case report with review of the literature. $J$ Neurosurg 1983;59:895-98.

7 Noetzel H. Arachnoidalcysten in der Cisterna ambiens. Zentralbl Neurochir 1940;5:281-94.

${ }^{8}$ Sweet WH. Spontaneous cerebral ventriculostium. Arch Neurol Psychiat 1940;44:532-40.

${ }^{9}$ Childe AE, McNaughton FL. Diverticula of the lateral ventricles extending into the cerebellar fossa. Arch Neurol Psychiat 1942;47:768-78.

${ }^{10}$ Macfarlane WV, Falconer MA. Diverticulum of the lateral ventricle extending into the posterior cranial fossa: report of a case successfully relieved by operation. J Neurol Neurosurg Psychiatry 1947;10:100-6.

"Perryman CR, Pendergrass EP. Herniation of the cerebral ventricles. Am J Roentgenol 1948;59:27-51.

${ }^{12}$ Kajtor F, Haberland K. Durch Hydrocephalus bedingtes Kammerdivertikel in der Cisterna ambiens. Arch Psychiat 1950;185:95-104.

${ }^{13}$ Tashiro K, Nakagawa T, Ito T, Tsuru M, Nakamura N. An autopsy case of von Recklinghausen's disease with aqueductal occlusion due to septum formation and gliosis, associated with subtentorial cyst formation. No To Shinkei 1975;27:333-42.

${ }^{14}$ Kapila A, Klingele TG, Slamovits TL, Burde RM, Ratcheson R, Gado MH. Quadrigeminal plate compression caused by a pulsion diverticulum. J Clin Neuro-ophthalmol 1981;1:135-40.

${ }^{15}$ Pennybacker J, Russell DS. Spontaneous ventricular rupture in hydrocephalus, with subtentorial cyst formation. J Neurol Psychiatry 1943;6:38-45. 